\title{
Making Chalk and Talk Accessible
}

\author{
S. Bennett \\ University of Hertfordshire \\ College Lane \\ Hatfield \\ +44(0)1707284342 \\ s.j.bennett@herts.ac.uk
}

\author{
J. Hewitt \\ University of Hertfordshire \\ College Lane \\ Hatfield, UK \\ +44(0)1707284327 \\ j.a.hewitt@herts.ac.uk
}

\author{
D. Kraithman \\ University of Hertfordshire \\ College Lane \\ Hatfield, UK \\ +44(0)1707285476 \\ d.a.kraithman@herts.ac.uk
}

\author{
C. Britton \\ University of Hertfordshire \\ College Lane \\ Hatfield UK \\ +44(0)1707284300 \\ c.britton@herts.ac.uk
}

\begin{abstract}
This paper investigates the development of an authoring package designed to mimic traditional "chalk and talk" delivery of content in education. It emphasizes the twin goals of making the output more accessible both for those with disabilities and for distance learners and also making the package usable by academic staff without requiring extensive training. It deals with issues arising from the capture of the material, the compromises and conflicts which are made in the satisfaction of accessibility guidelines and the implementation problems which arise. An authoring tool designed specifically for the production of accessible multimedia material is described as is preliminary work being undertaken to provide live subtitles of lectures.
\end{abstract}

\section{Categories and Subject Descriptors}

K.3.1 [Computer Uses in Education]: Distance Learning, Accessible Information

\section{General Terms}

Design, Human Factors

\section{Keywords}

Accessibility; authoring system; speech recognition; SMIL; XML

\section{INTRODUCTION}

The expression "chalk and talk" is often used disparagingly about non-technological approaches to education, and yet also articulates a well-used pedagogical method. The pedagogical richness of audio-visual teaching methods derives from two principal components. Firstly there is the fact of "imageargument synergy" as described by Diana Laurillard [1]. In her evaluation of educational media, she particularly praises television for its property of simultaneously showing the world and verbally articulating it.

"'Image-argument synergy' ties the experience (the image) to the description (the argument), synthesising

Permission to make digital or hard copies of all or part of this work for personal or classroom use is granted without fee provided that copies are not made or distributed for profit or commercial advantage and that copies bear this notice and the full citation on the first page. To copy otherwise, or republish, to post on servers or to redistribute to lists, requires prior specific permission and/or a fee.

CUU'03, November 10-11, 2003, Vancouver, British Columbia, Canada.

Copyright 2003 ACM 1-58113-701-X/03/0011...\$5.00. both levels of the academic discourse, and giving the students a 'vicarious conception', i.e. offering an insight into the way that the teacher thinks about the topic.'[1]

She intends this in the context of television, but we would argue that this synthesis of levels of discourse occurs in all audio-visual teaching scenarios. The second important value comes from what LaRose, Greg and Eastin called "teacher immediacy" [2]. Working from previous research that showed a positive correlation between student satisfaction and "instructor immediacy" (as measured in verbal or non-verbal behavior, the amount of anecdotal asides in lectures, the degree to which the instructor responded to student queries and exchanged observations with them), they showed that the audio presentation of material over the web, even if it lacked the video of the tutor's presence, could produce a sense of vicarious immediacy. They found that the simple recording of lectures and their replay over the web through an audio stream could prove to be a significantly more engaging medium than simple web pages [3]. In fact, it has been suggested elsewhere that students with cognitive impairments such as dyslexia benefit from multi sensory teaching methods [4].

There are however real difficulties in the digitization of a typical "chalk and talk" teaching scenario. Firstly the cost of producing televisual materials is high and generally not practical for an academic to undertake without considerable technical support. Secondly there are some real accessibility concerns: namely the difficulty of accessing the material thus produced by those with sensory impairments (hearing and sight). The true challenge therefore, of designing universally accessible teaching materials, is to both preserve the immediacy of the academic discourse while facilitating its digitization into accessible media.

The two principal obstacles in this regard are the paucity of software tools available to turn "chalk and talk" into digital form, and then the failure of those that do exist to make the content thus produced accessible. In the light of this, we at the University of Hertfordshire have been working on a number of related initiatives which attempt to "capture" the lecturing moment in order to reproduce it as a digital artifact. A significant outcome of these has been the creation of an authoring tool (SMIRK) for the purpose of producing accessible multimedia presentations by academics, useable without the need for technical assistance or extensive training. In another initiative we are adapting work on the production of subtitles by the use of speech recognition to produce live subtitles for lectures. 


\section{ACCESSIBILITY GUIDELINES}

Probably the most comprehensive and detailed set of recommendations for accessibility of web content is the draft of Version 2 of the Web Content Accessibility Guidelines published by the World Wide Web Consortium on $29^{\text {th }}$ April 2003 [5]. This is currently a draft document, and follows on from Version 1, produced in 1999, however it establishes very clear minimum standards against which documents can be tested, though some of them can be difficult to implement.

It establishes its recommendations under 5 main headings.

i. Perceivability

Content should be perceivable by all users: therefore images have alternate text descriptions, audio should be complemented by captioning, structure and appearance of documents remains separate

ii. Operability

All interface elements are operable by any users: e.g. duplicating mouse click commands with key strokes

iii. Navigability

Render the structure of the media clear to the users such that they are aware of where they are in the system

iv. Understandability

Write in clear English (or appropriate language)

v. Robustness

Use web technologies that are fully geared to working with accessibility technologies and user agents.

The summary here is a fairly succinct précis of the requirements of accessible documents, but even here it can seem daunting. How is the typical lecturer going to satisfy all these recommendations without vast amounts of extra work? Furthermore how can the typical lecturer remember them all?

If academics are unaided, the likelihood of them producing accessible content is small. However, if accessibility concerns could be incorporated into an authoring tool, a great load could be taken off the author's shoulders: for instance automatic provision of keyboard based navigation, or the tool inciting the creation of captions for slides.

\section{A TOOL FOR CAPTURING THE LECTURING MOMENT}

\subsection{Existing Tools}

A number of initiatives are around for converting Powerpoint presentations into something more accessible. There is the accessibility wizard developed by the University of Illinois [6] and captioning tools such as MAGpie[7]. However, PowerPoint is very much a tool geared to producing slides: its interface does not easily dispose it towards multimedia creation. There are facilities for adding audio to slides but these are squirreled away deep in its interface. Also, when exported, it is often tied to proprietary technologies which pre-suppose a Windows environment. As for tools like Flash and Director, whilst brilliant at multimedia creation, they require significant investment in time and effort for the user to be able to master their interfaces: probably too much time and effort for most academics. What is required is a tool for multimedia production whose methods are nearer those of the academic when lecturing to an audience.

One program used in the University of Hertfordshire which does this very well is Nzedsoft's AudioGraph [8] which is a tool specifically developed to produce very quick multimedia materials. The lecturer speaks and records pen movements alternately in order to produce what in the end will be an audio visual web presentation. This is then compacted into an AudioGraph presentation which achieves stunning levels of compression, such that presentations might even be viewable over a modem connection at $14.4 \mathrm{kbps}$ [9]

The great benefit of such presentations is their immediacy and expository power. However, AudioGraph's great strength in terms of performance, its fantastic compression rates, arises from what is its greatest weakness from the point of view of accessibility: it achieves its compression through the use of its own proprietary plug-in, rendering on-the-fly - its own aer AudioGraph presentations. Accessibility issues are not explicitly dealt with in its interface: for instance keyboard navigation through presentations, the provision of alternate text content for its graphics and captioning.

As a consequence, while seeking to emulate the naturalism of the AudioGraph authoring interface, we decided to produce a tool that would publish to more openly declared standards, and would seek to foreground much more explicitly accessibility principles in its core functionality. Also, AudioGraph being a predominantly Macintosh based authoring environment (its version 2.0 at the time of writing is only available on the Mac), we sought to produce a tool which would be based on the standards of the more commonly provided PC. The name of this tool is SMIRK (Simple Media-Integrating Resource Kreator).

\subsection{SMIRK}

In terms of its operation and user interface, SMIRK follows the style of a number of current slide presentation authoring tools. However, it is primarily an audio visual tool, so the recording console has high prominence at the top of the screen. The principle being that the author will produce the slide, and then give the narration so that the visual and audio parts of the slide will be linked thereafter. This gives the user the ability to produce the audio for the slide in more than one "take", permitting pausing and overwriting of already produced audio should it prove unsatisfactory. The SMIRK interface is shown in Figure 1.

On the top left there are the typical drawing and typing tools one expects: the rectangle, the ellipse, the arrow, the polygon, the polyline, particularly useful for people drawing things with graphics tablets. In this sense, SMIRK attempts to reproduce something of the annotation of an overhead transparency that might take place during a lecture. Using the polyline tool the author is able to circle and draw over items of particular interest in the underlying diagram as well as do handwriting.

There are explicit buttons "New Slide" and "Clone Slide". The purpose of the latter is again to reproduce a typical OHP strategy: namely the overlaying of one slide, or of cumulative pen strokes, upon the underlying diagram.

Finally, as one would expect there are the typical text tools; normal text and rich formatted text: the latter tool also allowing OLE objects such as graphs, organisation charts and equations to be inserted into the slide. 


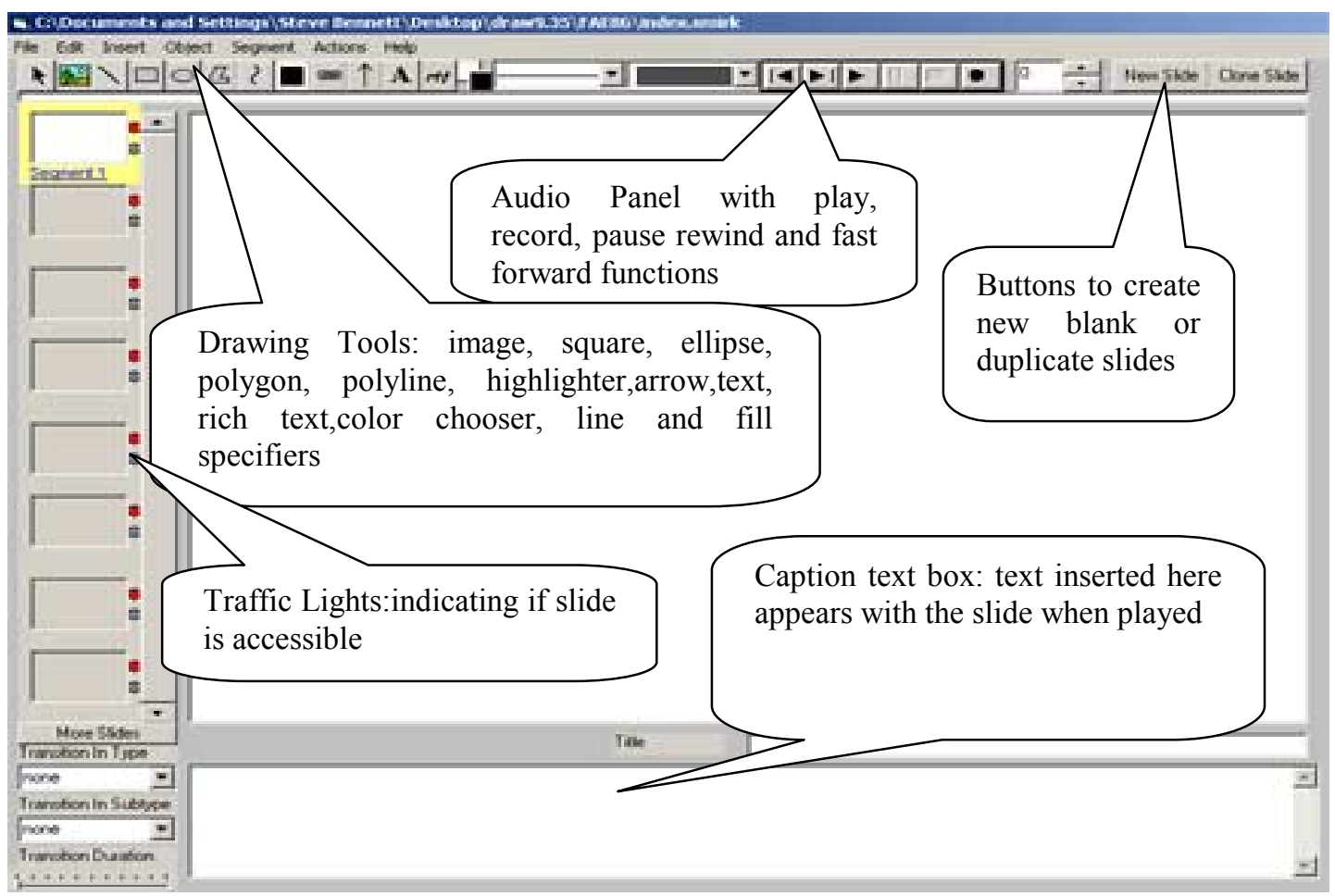

Figure 1. The SMIRK Interface

In the interface, accessibility features are fore-grounded from the start. The textbox at the bottom for the input of slide notes is permanently visible, and the red and green "traffic" lights at the side of the slide thumbnails indicate whether accompanying text has been inserted. This hopefully constitutes some kind of insistent appeal for accessibility awareness on the part of the author, however well disposed the individual is to satisfying it. The program also, in everything but the drawing operations, allows the user to substitute keyboard commands for mouse clicks, thus rendering the authoring interface controllable via voice commands making it as accessible as possible to the author.

At the end of the production of a slide sequence, the presentation is exported as a sequence of .jpg graphics and .mp3 audio files. The timing and sequencing of these files are then output in three time-dependent forms:

- SMIL 2.0 optimized for Real Player

- SMIL 1.0 optimized for QuickTime

- HTML+SMIL specifically for Internet Explorer 5.5 and above

The content is also output in two time independent forms:

- $\quad$ framed html with a table of contents in the left frame and content itself in the right: this allows for the easiest and least burdensome access to material, downloaded only as selected by the user.

- a single HTML document, containing all the accompanying text and graphics, ready to be printed out, an accessibility feature particularly appreciated by students everywhere.
In addition to the traffic lights at the side of the slide thumbnails, the other principal accessibility facilitator in the interface to the program is the accessibility audit screen produced immediately after the export command is triggered. This will check if all the slides have alternative caption text as well as unique titles. This facility is dealt with in greater depth in the section on accessibility certification.

\subsection{Multimedia XML Standards}

The most important standard in this area is indeed SMIL (Synchronized Multimedia Interchange Language) [10], though it is difficult to conceive of it as a single set of guidelines to which one should adhere but rather as a set of values which multimedia artifacts should embody. Besides its two core profiles (SMIL 2.0 the full spec. and SMIL Basic - mainly for PDAs), its influence has reached out into the co-option of many of its core modules into other XML languages, such as Microsoft's HTML+SMIL [11] and SVG (Scalable Vector Graphics) [12].

Its fundamental ethos is that multimedia should be programmed declaratively. Rather than using the procedural methods to govern how media plays, SMIL instead will describe the arrangement, both spatial and temporal of media, and give attributes to the media, suggesting when they can start and under what conditions, finish. There are other authoring systems for SMIL, but they are primarily used for aggregating previously developed media [13]. SMIRK on the other hand, both organizes and creates the media.

The great advantage of SMIL over proprietary or binary formats for multimedia, is the intense transparency of its descriptions, and the fact that they can be edited, like HTML, in a simple text editor without the need for elaborate authoring tools. In practice the great difficulty at this point is the variability of implementations, 
the vastness and modularization of the spec. making it very difficult to accurately foresee the behaviour in practice of the media sequences one creates in differing SMIL implementations.

\subsection{SMIL and Accessibility}

In accessibility terms SMIL presents some very powerful features, specifically the switch branching structure arising from specified system-test attributes. The most important of these are:

systemBitrate

systemCaptions

systemLanguage

systemAudioDesc

systemOverdubOrSubtitle

The SMIL client or user agent can be interrogated by the SMIL file, and consequences can be specified. This is done through the $<$ SWITCH $>$ tag. For example, if the systemLanguage of the client is French, then a multilingual SMIL file can specify that the French audio file is sent to the browser. If the client has "systemCaptions" set to "on", then additional captions will appear. Even a user preference of overdubbing as opposed to subtitles can be implemented for foreign language versions of SMIL files. An audio description of an image can be made to play if "systemAudioDesc" is "on". And depending on the value of the systemBitrate, different quality of media can be sent to the client [14]

SMIL also contains a number of standard accessibility features taken from other languages such as HTML, for instance the accessKey specifier which allows links to be activated by a user keypress rather than a mouseclick. Also there are alt and longdesc properties for media objects which function exactly as they do in HTML (offering respectively a short description, or a link to a url containing a long description of the media).

However, as with all production, the specification itself cannot guarantee the production of accessible materials: that depends on the authors. Any form of multimedia which involves interactivity as well as temporality of media can produce potential accessibility problems: if a link has a duration of 10 seconds, but a user does not click within its active period, the navigational possibility will be lost, thus severely disadvantaging someone who requires greater time to inspect the material. SMIRK therefore creates a special page of HTML for each presentation containing all the external links shown during the presentation. This allows the user to view it even if they have missed clicking on a link while it was alive.

In all the dynamic XML forms that it attempts to output, SMIRK gives the user the ability to navigate though the material and replay various slides, both through mouse events and through "access Keys" (if they are implemented in the client). Therefore, SMIRK attempts to do as much as possible in its power to make it easy for the lecturer to produce accessible audio visual presentations to be delivered over the internet. However, considerable work still needs to be done to make this more effective.

\subsection{Conflicts and Compromises}

SMIRK does not yet, and might never, produce universally accessible content. Reasons for this can be broken down into a number of areas: the current level of development of SMIRK, the implementations and specification of SMIL itself and finally the slightly intangible quasi-philosophical issues arising from conflicts between differing accessibility needs.

\subsubsection{SMIRK development}

Currently SMIRK exports all its visual media as JPEGS. If this were not viewable by the user, it is hoped the author would have filled in the caption box on each slide such that the textual substitution would appear to help the user. The audio voice over also should compensate for its absence.

However, outputting the graphics to JPEG (a fixed raster based graphical format) is not a good idea, and a vector based graphical format would be more desirable: the reason specifically is to permit the users to magnify images, facilities easily done within Flash's .swf binary format, and in SVG (Scalable Vector Graphics). The advantage of Flash here is its ubiquity and reliability. However, SVG offers some features which have greater accessibility potential. For example, because it is a text based format, it allows searching within it - that search feature is not available within Flash.

More interesting for us is the potential for using Style Sheets. SVG has the same ability to override its default rendering to the user through a style sheet that HTML has. This could have vast potential for users with varying degrees of color blindness. Instead of looking at a graphic with a pre-specified set of colours, the style sheet could change colours around and perform various accentuations of the graphic in order to facilitate a better viewing by a user thus impaired [15]. It is part of the current development plan for SMIRK that outputting of visual media to SVG and Flash be possible in the next release. SMIRK contains internal vector representations of its graphical objects and this will be exportable to SVG as text and to the binary .swf format through the SourceForge Bukoo libraries [16].

Similar issues arise in fields like equations and organisational charts. One can easily use the Microsoft Equation Editor in SMIRK to produce the graphical version of an equation, yet this is not accessible. Being able to put MathML within a SMIL file would help here (yet there are issues regarding the implementation of other XML languages within SMIL clients).

\subsubsection{SMIL Specification and Implementation}

Certain issues arise from SMIL implementations. For instance the QuickTime player implements only partially the SMIL 1.0 specification [17]. One of the problems arising from this is the inability to do internal hyperlinking, thus allowing the user to easily "seek" through a multimedia presentation in order to only play specified parts. It compensates for this by allowing "chapters" to be defined by media containers (an extension to the language) which is navigable via the QuickTime interface, though this is not to our knowledge controllable by keyboard input.

Other issues relate to implementation. At the time of writing, Internet Explorer's HTML+SMIL did implement Access Key correctly, whereas Real Player (version 6.0.10.505) did not appear to implement it at all.

Even if Access Key was implemented correctly, it is not a particularly elegant solution to media navigation. The most common paradigm of media navigation we currently have is 
probably the TV remote control. In situations of at least 100 channels there are usually methods of keying in large numbers and having the television navigate straight there. The access key by definition can be only one character at a time. Some more elaborate way of accommodating keyboard input would be highly desirable within SMIL.

\subsubsection{General Issues for All-Round Accessibility}

This area is perhaps the most problematic. Most of the well adopted and implemented accessibility features of modern XML languages (for instance ALT and LONGDESC) began in the era of static html. In that context, giving alternative text for images is an absolutely essential feature.

In the context of a timed audio visual presentation however, we can anticipate that the spoken narration will describe the image to a certain extent. However, its major purpose is not so much description as interpretation, the concentration on certain aspects of the image in order to further reveal its meaning. Following accessibility guidelines fully would urge us to provide therefore both a LONGDESC of the image, as well as the audio voice-over. And yet the voice-over itself is predicated on the simultaneous presence of the visual image. In this context would a highly visually descriptive LONGDESC of the image followed by a more interpretative voice-over be an acceptable reproduction of the multi-sensory exposition?

In the case of the user with auditory impairment we might imagine that refiguring the simultaneity of the voice-over, in this case with subtitles or captioning would be more faithful to the original experience. However, any attempt at reproducing multi-sensory simultaneity across one sense can be problematic as the new Web Accessibility guidelines make clear:

"Where possible, provide content so that it does not require tracking multiple simultaneous events with the same sense, or give the user the ability to freeze the video so that captions can be read without missing the video" [18].

Other issues arise when applying accessibility techniques such as text-to-speech to audio-visual material For instance, how would such software, which is used to read static web pages, interact with ones whose purpose is to disseminate audio. How would additional audio information provided with images to be used when systemAudioDesc system-test attribute is "on", interact with the piece of audio that the SMIL file is broadcasting at that moment?

\subsubsection{Accessibility Certification: the Spirit and the Letter}

As mentioned above, SMIRK exports to three time based formats (SMIL 1.0, SMIL 2.0 and XHTML+SMIL) as well as two time independent formats (framed and non-framed HTML). In these two latter formats, there is a real test that can be applied to the outputs to verify accessibility guideline conformance, namely the Bobby application produced by the Watchfire Corporation[19], which tests for conformance to the Web Content Accessibility Guidelines[20] and Section 508 of the U.S Rehabilitation Act[21].

Both the framed and the non framed HTML produced by SMIRK is compliant with the above guidelines and can therefore by used with the "Bobby Approved" logo[22]. The only caveat being that the author must make sure that all the slides in the presentation have unique titles.

This however, is facilitated by the accessibility audit screen which is produced after any export in the program.

\begin{tabular}{|c|c|c|}
\hline 9. Access & sibility Report & - \\
\hline Slide & Captions Text & Slide Title \\
\hline Slide1 & No Captions & $\mathrm{OK}$ \\
\hline Slide2 & No Captions & $\mathrm{OK}$ \\
\hline Slide 3 & No Captions & $\mathrm{OK}$ \\
\hline Slide4 & No Captions & $\mathrm{OK}$ \\
\hline Slide 5 & No Captions & $\mathrm{OK}$ \\
\hline Slide6 & No Captions & $\mathrm{OK}$ \\
\hline Slide ? & No Captions & $\mathrm{OK}$ \\
\hline Slide8 & No Captions & Duplicate Slide Title [1] with slide 1 \\
\hline
\end{tabular}

At this point a quick check shows if the slides have captions, and if they have titles, and moreover, if those titles are unique. The reason for this insistence on the uniqueness of slide titles, is that in the non timed based HTML pages, those slide titles will become internal links within the pages, and one particular accessibility guideline is that the same link text should not appear in a web page, if the addresses pointed to by those links are not identical.

This requirement, of the author to produce unique titles for slides, shows that the program cannot absolutely guarantee accessibility compliance by software methods alone, but relies on the author to observe the rules too. But it also points to the fact that the formal validation of guidelines compliance is never enough. The author for instance could put in a simple space on all of the caption boxes, and thus be formally, guidelines compliant and yet in no way satisfying any accessibility needs that a user might have. Less dramatically, the caption text might not be as meaningful or specific as the voice-over itself. However, as yet, there is no computerized method of evaluating the fidelity and meaningfulness of the captions thus produced. Thus, while adherence to the letter of accessibility guidelines can be encouraged, and to a certain extent, enforced, adherence to the spirit of them, that is to say of universal access to the materials by all users, depends on the conscientiousness and thoroughness of the author.

\subsection{Capturing and Captioning}

Ultimately the requirement of SMIRK is to allow the lecturer to produce accessible material with as little repurposing and duplicating (what we might term extra work) as possible. However, in the case of the written notes/captions, some duplication is indeed already taking place: the lecturer effectively writes what she is speaking. Ideally the program itself could write what the lecturer is speaking through techniques such as speech recognition. And more concretely, would do so in a timed and recorded way, in order to enable the provision of captions reproducing the timing of the spoken narration. We are currently 
seeking to incorporate speech recognition functionality into SMIRK, building on research already done at the University of Hertfordshire.

\section{SPEECH RECOGNITION TO AID ACCESSIBILITY}

Speech recognition software has finally reached a level of accuracy where it offers a viable alternative to text-based input. Two products currently dominate the field for general-purpose speech-to-text applications, these are IBM's ViaVoice and Dragon's Naturally Speaking, and recognition rates of approaching $100 \%$ can be achieved by trained speakers operating in domains where there are few out-of-vocabulary words and consistent noise levels. Much greater challenges are presented by the transmission of live speech, by the capture of speech from untrained or poorly trained speakers and by speech recognition in noisy environments. All of these challenges are being addressed by researchers, including a team at the University of Hertfordshire.

\subsection{Transmission of live speech}

The Subspeak project ${ }^{1}$ carried out by a team from the University of Hertfordshire in partnership with a company which produces subtitling equipment, has developed software which allows the subtitling of live television programmes. This software is now in use by two major UK television companies and one company has reported accuracy rates of $98 \%$ for sports subtitling without the need to edit any of the subtitles before they are shown on air. The system uses the IBM ViaVoice recognition software and is used by trained speakers operating from a quiet television studio. An acceptable level of accuracy is obtained only by pre-loading the recogniser with any possible out-of-vocabulary words before the live broadcast. Typical out-of-vocabulary words would include the names of players in a football team or technical terms in specialist domains. Customised language models or "Topics" can be built for specific domains using the ViaVoice Topic Factory and tests have shown improvements in recognition rates of between $1 \%$ and $3 \%$ due to their use [23].

\subsection{Capturing speech from a live performance}

There are further problem areas to be addressed when trying to subtitle a live performance (for example a lecture) [24] Experiments at UH have highlighted some of the difficulties, for example:

- $\quad$ Each performer (lecturer) will have to find time to train the system, and experience shows that not all speakers find it easy to achieve acceptable recognition rates [25]

- Mobile equipment is used and there is little time to finetune it before a performance to adjust to the noise levels

- A way must be found to input punctuation without speaking it

- Software is needed to output the subtitles in conjunction with a presentation (e.g. PowerPoint)

${ }^{1}$ LINK project GR/M15958 under the Broadcast Technology Initiative with the University of Hertfordshire and Synapsis Ltd.
In preliminary trials at $\mathrm{UH}$, speakers who had consistently achieved a rate of $98 \%$ in a quiet environment managed to achieve only $90 \%$ in a classroom situation and further work is being undertaken to address the problems identified above. The Liberated Learning project [26] reports successful trials in classroom subtitling at a number of institutions but does not give any recognition rates.

A new tool, Lecturepad, has been developed at UH which can be used in conjunction with PowerPoint to provide live subtitles during a slide show. Punctuation can be configured to appear when delays of a pre-determined length occur in the input stream (for example 250msecs for a comma and 500 msecs for a fullstop). The tool is being fine-tuned to meet the demands of different types of PowerPoint presentation prior to being tested in a classroom.

The obvious advantage of subtitling live performance is to provide text for hearing impaired listeners and for those who may not have a complete understanding of the language in which the performance is given. A further advantage is that a text transcript of the performance can be produced. This can be further edited and made available to the audience.

\subsection{Recorded Speech Recognition}

In the context of the SMIRK program, there would be advantages in providing a tool to allow captions to be created by speech. The advantage of creating the captions 'off-line' is that the errors in transcription can be resolved, and in resolving them the recognition engine can be further enhanced. Currently existing speech recognition products however do not include timing information about the speech transcribed, which is essential if one is to provide accurate captioning, and specifically, the imageargument synergy mentioned earlier, the dance of meaning between the mutating image and its meta-narration. It is an ambition of the SMIRK tool to incorporate some form of timed speech recognition into its core functionality in order to provide such captioning.

\section{CONCLUSIONS}

This paper has identified some of the complex trade offs to be made when attempting to produce accessible materials. However, what our work has shown is that only through embedding accessibility facilitation within the normal routines of teaching can the goal of widely available accessible learning content be achieved.

\subsection{Future Work}

The SMIRK tool was released for evaluation on the $7^{\text {th }}$ July 2003[27]. Intended applications are a 40 lesson course on web programming in Computer Science and an extensive economics glossary for the University of Hertfordshire Business School. Take up and user satisfaction when using these resources will be evaluated from web server logs and questionnaires of the students who use them.

A further experiment envisaged is to get students themselves to use SMIRK in order to peer-evaluate web based applications produced by other students. The purpose of this is to create a context in which students can apply usability principles to real development scenarios. This use, the exploitation of SMIRK by 
students themselves might prove an even more fruitful area of activity than the broadcasting use of the tool envisaged by this paper.

In technical terms, immediate future developments include the addition of speech recognition into the program so as to be able to generate timed sub-titles without requiring any explicit scheduling by the author. The other important goal is exporting the visual material to SVG so as to facilitate differential viewing of the graphics through style-sheets.

\section{REFERENCES}

[1] Laurillard D, Rethinking University Teaching $2^{\text {nd }}$ Edition, RoutledgeFalmer 2002 p102

[2] LaRose,Gregg,Eastlin, Audiographic Telecourses for the Web: An Experiment http://www.ascusc.org/jcmc/vol4/issue2/larose.html

[3] Ibid

[4] University of Sheffield: Teaching Students with Dyslexia: 9. Teaching Strategies. http://www.shef.ac.uk/disability/teaching/dyslexia/9_strategi es.html

[5] Caldwell, Chisholm, White, Vanderheim. Web Content Accessibility Guidelines 2.0 http://www.w3.org/TR/WCAG20/.

[6] University of Illinois Microsoft Office Web Publishing Accessibility Wizard. 2003. http://www.rehab.uiuc.edu.office.

[7] National Centre for Accessible Media Access generator (MAGpie). 2003. http://ncam.wgbh.org/webaccess/magpie

[8] Nzedsoft Audiograph Homepage 2003. http://www.nzedsoft.com/audiographhomepa.html.

[9] Jessop, C., Shafarenko, A., and Slusanschi, H. Lowbandwidth, multimedia tools for web-based lecture publishing. 1999. http://www.nzedsoft.com/Papers/iee.pdf

[10] W3C (2001) Synchronized Multimedia Integration Language (SMIL 2.0). http://www.w3.org/TR/smil20/

[11] W3C(2002) XHTML+SMIL Profile. 2003. http://www.w3.org/TR/XHTMLplusSMIL/
[12] W3C(2003) Scalable Vector Graphics (SVG) 1.1 Specification. http://www.w3.org/TR/SVG11

[13] See the Grins Editor developed by Oratrix(2002) Grins for SMIL Homepage. http://www.oratrix.com

[14] W3C(2001). ContentControlIMS-PredefinedSystemTest http://www.w3.org/TR/smil20/smil-content.html

[15] Adobe Systems Incorporated Tutorial - Cascadiing Style Sheets. 2000. http://www.adobe.com/svg/basics/css.html

[16] Sun (2001) About Bukoo. http://bukoo.sourceforge.net

[17] Apple Corporation (2003). Apple - Products - QuickTime and SMIL. http://www.apple.com/quicktime/authoring/qtsmil.html

[18] Caldwell, B., Chisholm,W., White,J., Vanderheim,G. 2003. http://www.w3.org/TR/WCAG20/

[19] Watchfire Corporation, Welcome to Bobby Worldwide, http://bobby.watchfire.com/bobby/html/en/index.jsp

[20] Caldwell, Chisholm, White, Vanderheim. Web Content Accessibility Guidelines 2.0 http://www.w3.org/TR/WCAG20/.

[21] The Access Board, The Rehabilitation Act Amendments, http://www.access-board.gov/sec508/guide/act.htm

[22] Watchfire Corporation, Icon Guidelines. http://bobby.watchfire.com/bobby/html/en/icon.jsp

[23] Hewitt, J., Lyon, C., Ahmed, R., Lambourne, A., Speech Based Subtitles for Live Performance. Accepted for HCI International 2003, Crete, June 2003.

[24] Ibid

[25] Hewitt, J., Bateman, A., Lambourne, A., Nobay, K., Hewitt, R., Subtitles from Simultaneous Transdiction: Multi-Modal Interfaces for Generating and Correcting Real-Time Subtitles. Proc. Of HCI International 2001.

[26] Bain, K., Basson, S.H., Wald, M., (2002). Speech Recognition in University Classrooms: Liberated Learning Project. Assets 2002, July 8-10, 2002 Edinburgh, Scotland.(pp192-196)

[27] Download from http://homepages.feis.herts.ac.uk/smirk 